$\begin{array}{cc}\text { Programa de Pós-Graduação em Engenharia de Produção - PPGEP } & \\ \text { Laboratório de Qualidade de Vida - LaQVida } & \text { REVISTA BRASILEIRA DE } \\ \text { Universidade Tecnológica Federal do Paraná - UTFPR } & \text { QUALIDADE DE VIDA } \\ \text { Ponta Grossa - PR - Brasil } & \text { Q7. n. 02, abr./jun. 2015, p. 56-64 }\end{array}$

DOI: $10.3895 /$ rbqv.v7n2.2724

\title{
Percepção e nível de qualidade de vida entre pré-escolares
}

\section{Perception and level of quality of life among preschool}

\author{
Angélica Patrícia Pereira \\ Universidade do Contestado - UnC - Mafra - Santa Catarina - Brasil \\ angelica_patricia100@hotmail.com \\ Daniel Rogério Petreça \\ Universidade do Contestado - UnC - Mafra - Santa Catarina - Brasil \\ profdaniel@unc.br
}

\section{RESUMO}

OBJETIVO: Analisar a qualidade de vida de pré-escolares de uma escola pública do Município de Piên - PR identificando os níveis de percepção individual e coletiva.

MÉTODOS: O estudo caracteriza-se de natureza observacional, quantitativo, pelo procedimento de levantamento (Survey). A amostra foi composta por 50 pré-escolares com média de idade de 5,6 $(\mathrm{DP}=0,3)$ anos. Para mensuração da qualidade de vida foi utilizado o questionário Autoquestionnaire Qualité de Vie Enfant Imagé (AUQEI). Os dados foram armazenados no programa Microsoft Office Excel, versão 2010, e tratados no Programa Estatístico SPSS 20.0 por meio de estatística descritiva, indutiva e de frequência.

RESULTADOS: Verificou-se que a percepção de qualidade de vida dos pré-escolares foi satisfatória $(58,5 \%)$. Ao comparar meninos e meninas percebe-se que os meninos apresentaram um escore satisfatório maior (65,0\% contra 47,6\% das meninas). Foi possível verificar que os domínios mais relevantes para a qualidade de vida referem-se ao lazer e à família.

CONCLUSÕES: A qualidade de vida dos pré-escolares envolvidos no estudo apresenta-se satisfatória.

PALAVRAS-CHAVE: Qualidade de vida. Pré-escolares. Crianças.

\footnotetext{
ABSTRACT

OBJECTIVES: This study aimed to analyze the quality of life of preschool children in a public school in Piên city, located at Paraná state, in Brazil.

METHODS: The study characterized the observational nature, quantitative characteristics, using the survey procedure. The sample consisted of 50 preschool children with a mean age of 5.6 $(\mathrm{SD}=0,3)$ years. For measuring quality of life the study used the questionnaire AUQEI (Autoquestionnaire Qualité de Vie Enfant Image). Data were stored in Microsoft Office Excel, version 2010 program and treated in the statistical program SPSS 20,0 using descriptive, inductive and frequency statistics.

RESULTS: It was found that the perception of quality of life of preschool children was satisfactory $(58,5 \%)$, when comparing boys and girls realizes that the boys had a higher satisfactory scores
} 
(65,0\% versus $47,6 \%$ of girls). We found that the most relevant areas for quality of life refers to leisure and family.

CONCLUSIONS: The quality of life of preschool children involved in the study presents satisfactory.

Keywords: Quality of life. Preschoolers. Children.

\section{Introdução}

Qualidade de vida (QV) pode ter significados diferentes ao longo da vida, considerando assim sensações vividas e interpretadas nos domínios físico, psicológico, relações sociais e meio ambiente. Atualmente existe um interesse no conceito de QV tanto no meio acadêmico, quanto pelos profissionais da área da saúde (GORDIA et al., 2010), particularmente no que diz respeito à sua avaliação ou mensuração, quer individual ou coletivamente (LAURENTI, 2003).

Apesar da sua concepção subjetiva e de difícil quantificação, variando suas definições de acordo com os interesses do indivíduo, de seu grupo cultural e de seus próprios valores (ASSUMPÇÃO JR. et al., 2000), a avaliação da QV é, na maioria das vezes, mensurada por questionários (CICONELLI et al. 1999).

Avaliar a QV de crianças torna-se cada vez mais importante (SOARES et al., 2011). Segundo Gaspar et al. (2006), apesar da mensuração ser complexa, na avaliação da QV da criança e do adolescente, torna-se fundamental ter em conta a experiência subjetiva em vez das condições de vida.

Ao verificar estudos que analisam a percepção da QV de crianças, foi identificado maior concentração na área pediátrica, como no estudo de Barreire et al. (2003) com crianças ostomizadas, de Elias (2005) com portadores de Transtornos Invasivos do Desenvolvimento, e de Ferreira, D. C. (2005) em um grupo de crianças infectadas pelo HIV, sendo que, segundo Fialho (2006) ainda são insuficientes estudos no contexto escolar, de modo específico no Brasil.

Para tanto, a escola pode ser um canal de acesso a estratégias para a promoção de saúde por meio de atividades de ensino e de vivências, sendo fundamental a identificação de necessidades, demandas e percepções da criança em relação à QV, obtendo assim, dados relevantes na implementação de intervenções

Com base nessa perspectiva, o presente estudo teve por objetivo analisar a QV de préescolares de uma escola pública do município de Piên - PR identificando os níveis de percepção individual e coletiva.

\section{Metodologia}

O presente estudo adotou uma abordagem de natureza observacional, quantitativa, pelo método de procedimento de levantamento (Survey), sendo conduzido com pré-escolares de uma escola pública de Piên, município localizado na região sudeste do estado do Paraná de 11.236 habitantes.

A escola selecionada foi de caráter intencional e possui 60 crianças matriculadas na préescola. Como critérios de inclusão na amostra foram estabelecidos: estar devidamente matriculado, frequentando regularmente e expressar vontade de participar da pesquisa. Como critérios de exclusão foi utilizado apenas o fato da não permissão dos pais/responsáveis para a participação neste estudo. Para início da pesquisa foi enviado aos pais ou responsáveis das crianças o Termo de Consentimento Livre e Esclarecido, onde se encontravam todas as informações referentes ao estudo realizado. O período da coleta de dados com as crianças ocorreu no mês de março de 2014, e foi 
realizada nas dependências da própria escola, durante as aulas de Educação Física em entrevista individual.

Com a finalidade de obter informações sobre o perfil socioeconômico foi aplicado aos pais/responsáveis um questionário com sete questões de múltipla escolha, com perguntas referentes à renda familiar, nível de escolaridade dos pais, quantidade de pessoas que moram na casa, situação da residência (alugada, cedida ou própria) e localização da moradia (zona rural ou urbana).

Para mensuração do nível de QV dos pré-escolares foi aplicado o Autoquestionnaire Qualité de Vie Enfant Imagé (AUQEI) (ASSUMPÇÃO JR. et al., 2000). Este instrumento é composto por 26 questões objetivas (Quadro 1), com 4 opções de resposta - muito infeliz, infeliz, feliz e muito feliz - que equivalem a um escore de satisfação que varia de 0 a 3 , respectivamente.

Quadro $1-$ Questões Autoquestionnaire Qualité de Vie Enfant Imagé (AUQEI)
\begin{tabular}{|l|}
\hline \multicolumn{1}{|c|}{ Diga como você se sente } \\
\hline 1. À mesa, junto com sua família \\
\hline 2. À noite, quando você se deita \\
\hline 3. Se você tem irmãos, quando brinca com eles \\
\hline 4. Á noite, ao dormir \\
\hline 5. Na sala de aula \\
\hline 6. Quando você vê uma foto sua \\
\hline 7. Em momentos de brincadeiras, durante o recreio escolar \\
\hline 8. Quando você vai a uma consulta médica \\
\hline 9. Quando você prática um esporte \\
\hline 10. Quando você pensa em seu pai \\
\hline 11. No dia de seu aniversário \\
\hline 12. Quando você faz as lições de casa \\
\hline 13. Quando você pensa em sua mãe \\
\hline 14. Quando você fica internado no hospital \\
\hline 15. Quando você brinca sozinho \\
\hline 16. Quando seu pai e sua mãe falam de você \\
\hline 17. Quando você dorme fora de casa \\
\hline 18. Quando alguém te pede que mostre alguma coisa que você sabe fazer \\
\hline 19. Quando amigos falam de você \\
\hline 20. Quando você toma remédios \\
\hline 21. Durante as férias \\
\hline 22. Quando você pensa em quando estiver crescido \\
\hline 23. Quando você está longe de sua família \\
\hline 24. Quando você recebe as notas da escola \\
\hline 25. Quando você está com seus avós \\
\hline 26. Quando você assiste televisão \\
\hline
\end{tabular}

Fonte: Adaptado de Magnificat e Dazort (1997 apud ASSUMPÇÃO JR. et al., 2000).

Entre as 26 questões, 18 estão contidas em 4 domínios:

a) Função (questões: $1 ; 2 ; 4 ; 5 ; 8$ );

b) Família (questões: $3 ; 10 ; 13 ; 16 ; 18$ );

c) Lazer (questões: $11 ; 21 ; 25$ );

d) Autonomia (questões: $15 ; 17 ; 19 ; 23 ; 24$ ).

As questões $6 ; 7 ; 9 ; 12 ; 14 ; 20 ; 22 ; 26$ não pertencem a um domínio específico sendo analisadas de forma isolada com o escore obtido.

Após o preenchimento dos questionários, seu somatório pode variar de 0 a 78 pontos. Neste estudo foi utilizado o ponto de corte de 48 pontos para avaliar o pré-escolar em nível de QV satisfatório e não satisfatório, proposto por Assumpção Jr. et al. (2000).

Para aplicação do instrumento, cada questão foi apresentada pelo avaliador, sendo solicitado ao avaliado a escolha por meio das faces do AUQEI. Antes da entrevista, solicitou-se às crianças 
que relatassem uma experiência que tenham vivido para cada imagem representativa, o que permite compreender a situação e expressar os seus próprios sentimentos.

Figura 1- Faces representativas do questionário AUQEI

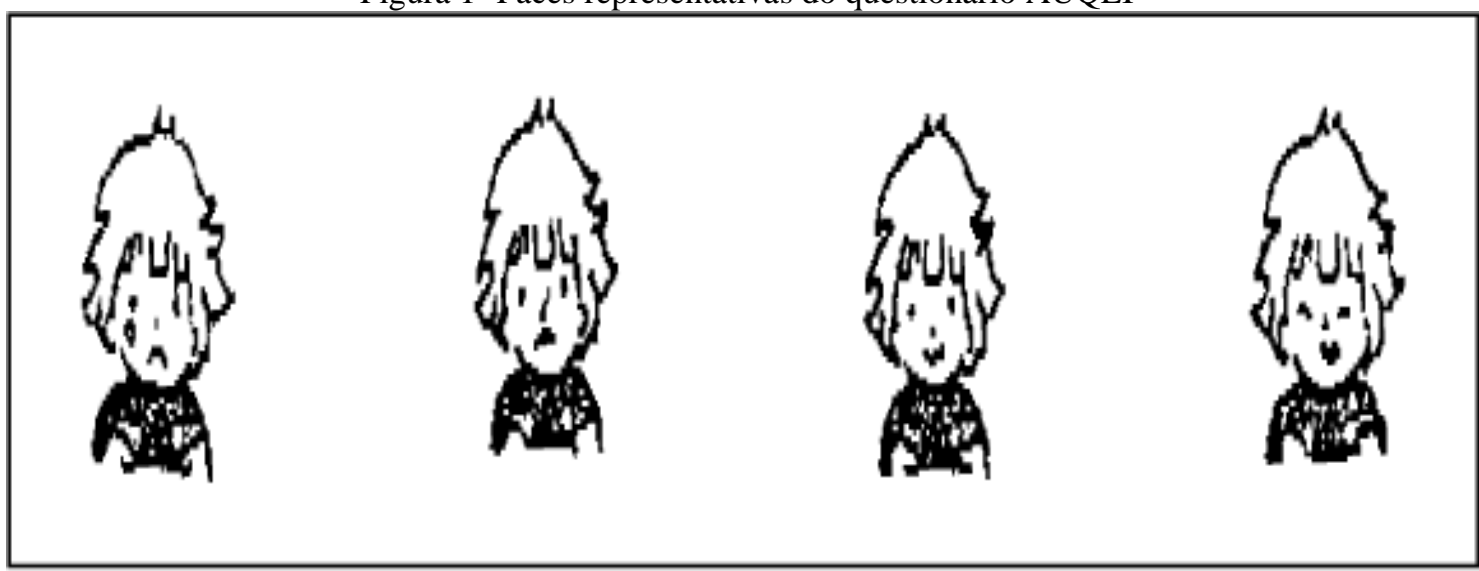

Fonte: Magnificat e Dazort (1997).

Os dados foram armazenados no programa Microsoft Office Excel, versão 2010. Para o tratamento dos dados foi utilizado o Programa Estatístico SPSS 20.0 sendo desenvolvida estatística descritiva e inferencial.

\section{Resultados}

Fizeram parte deste estudo 50 pré-escolares, sendo $29(58,0 \%)$ meninos e 21 meninas $(42,0 \%)$, com uma média de idade de 5,6 $(\mathrm{DP}=0,3)$ anos. A Tabela 1 apresenta os dados obtidos com o questionário sociodemográfico, realizado com os pais/responsáveis.

Tabela 1 - Questionário sócio demográfico, realizado com os pais/responsáveis dos pré-escolares pesquisados

\begin{tabular}{|c|c|}
\hline Quesito avaliado & Frequência relativa \\
\hline \multicolumn{2}{|l|}{ Moradia } \\
\hline 1 a 3 pessoas & 24 \\
\hline 4 a 7 pessoas & 70 \\
\hline 8 a 10 pessoas & 4 \\
\hline Mais de 10 pessoas & 2 \\
\hline \multicolumn{2}{|l|}{ Residência } \\
\hline Própria & 76 \\
\hline Alugada & 20 \\
\hline Cedida & 4 \\
\hline \multicolumn{2}{|l|}{ Local } \\
\hline Rural & 24 \\
\hline Urbano & 76 \\
\hline \multicolumn{2}{|l|}{ Escolaridade dos pais } \\
\hline Ensino Fundamental & 44 \\
\hline Ensino Médio & 38 \\
\hline Ensino Superior & 10 \\
\hline Pós-graduação & 8 \\
\hline \multicolumn{2}{|l|}{ Renda familiar } \\
\hline Até 1 salário mínimo & 20 \\
\hline De 1 a 3 salários mínimos & 54 \\
\hline De 4 a 6 salários mínimos & 20 \\
\hline Mais de 6 salários mínimos & 6 \\
\hline
\end{tabular}

Fonte: Autoria própria (2014).

R. bras. Qual. Vida, Ponta Grossa, v. 7, n. 2, p. 56-64, abr./jun. 2015. 
O Gráfico 1 apresenta a pontuação do nível de QV por meio do AUQEI dos pré-escolares obtidos pela análise descritiva de média das respostas de forma isolada, divididos pelo sexo. Para tanto se obteve valores muito próximos entre os sexos, obtendo apenas diferença estatística significativa nas questões 11 e 13.

Gráfico 1 - Apresentação da pontuação do nível de QV de forma isolada e divididos por sexo dos pré-escolares

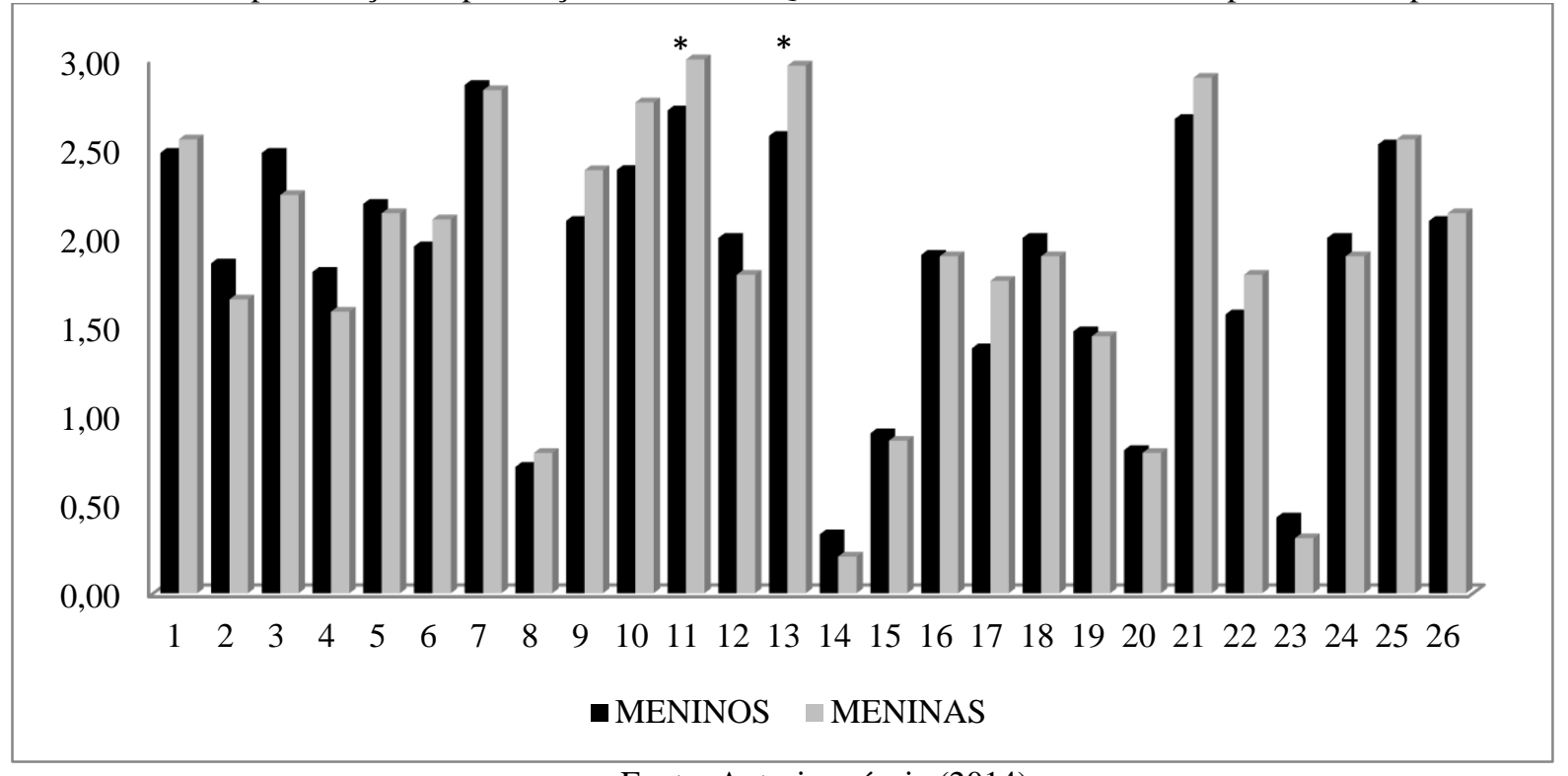

Fonte: Autoria própria (2014).

Obs: * significante pela análise Mann-Whitney.

O Gráfico 2 apresenta a média dos escores divididos por sexo em relação aos quatro domínios do AUQEI.

Gráfico 2 -. Escores divididos por sexo nos quatro domínios do AUQEI dos pré-escolares

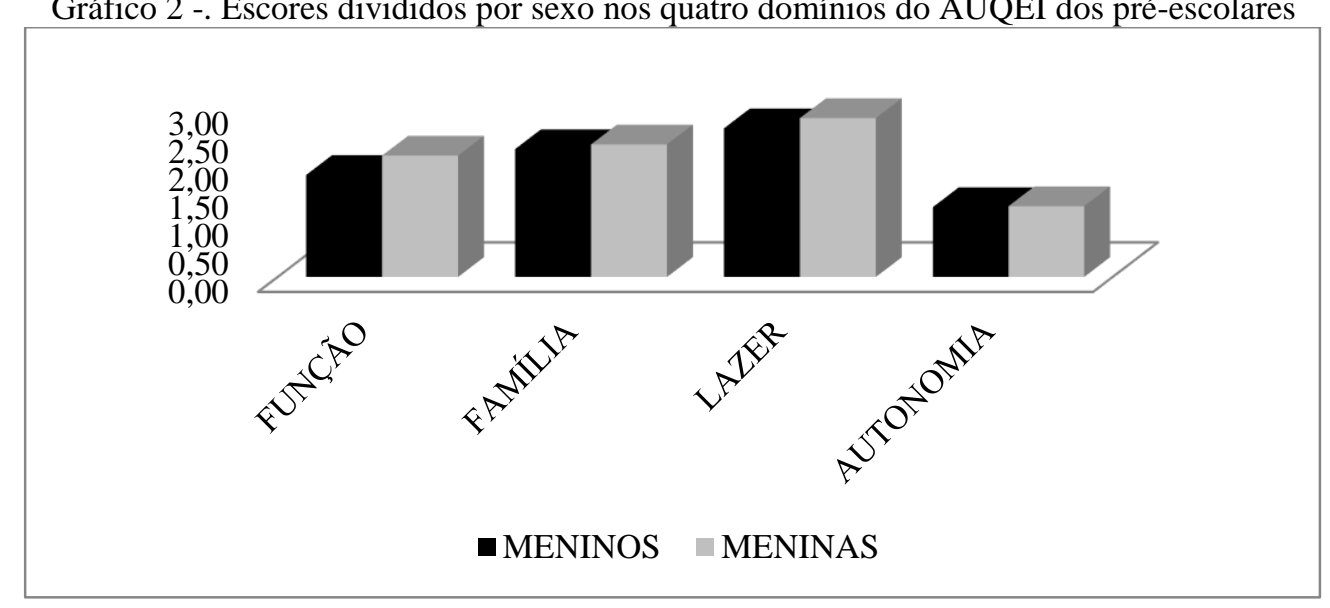

Fonte: Autoria própria (2014).

O Gráfico 3 apresenta o somatório das respostas classificadas de acordo com o ponto de corte sugerido por Assumpção Jr. et al. (2000) demonstrando situação 'favorável' ou 'desfavorável'. 
Gráfico 3- Distribuição de frequência em relação à percepção de QV dos pré-escolares

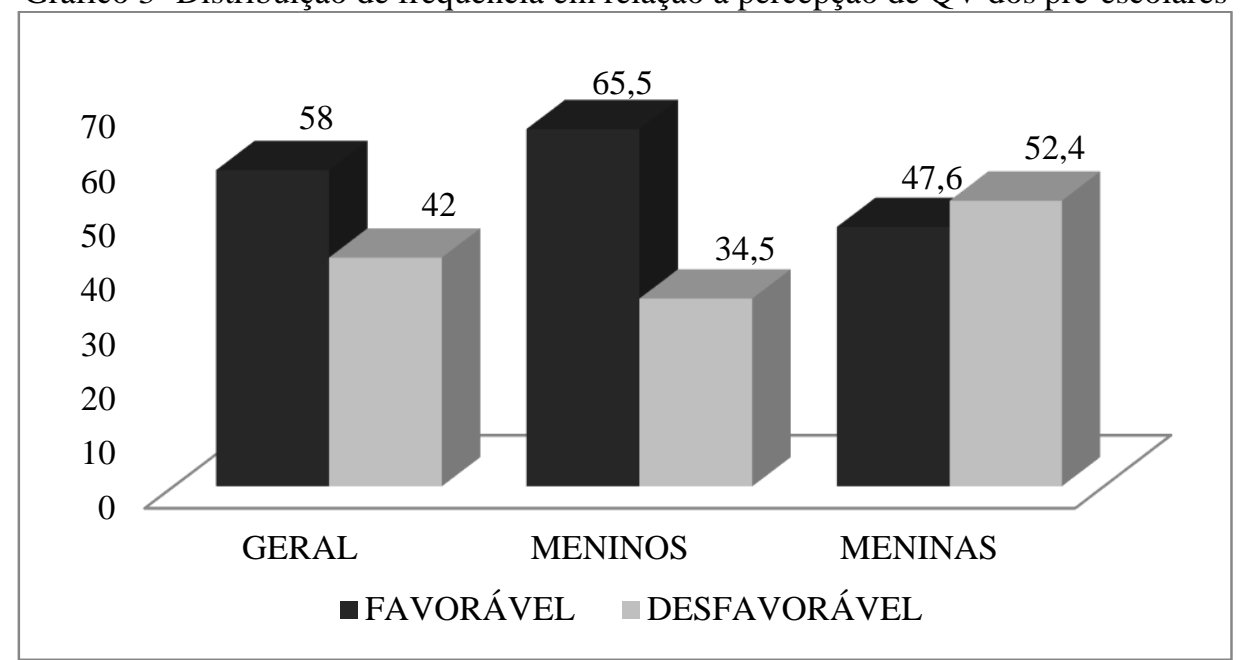

Fonte: Autoria própria (2014).

\section{Discussão}

A avaliação da QV em crianças foi inicialmente realizada pela percepção dos pais, considerando-se que crianças seriam incapazes de realizar tal tarefa (SOUZA et al., 2014). Para tanto, segundo Connolly e Johnson (1999) foram desenvolvidos instrumentos de mensuração da QV estabelecendo a criança como sujeito de pesquisa, levando em conta a sua capacidade e seus limites no processo complexo relacionado à $\mathrm{QV}$, estimando-se que entre quatro e seis anos, é a faixa etária onde elas têm o discernimento e a capacidade de relatar os domínios mais concretos de sua percepção (CONNOLLY; JOHNSON, 1999).

Ao verificar a percepção individual de QV dos pré-escolares deste estudo, as questões com maiores escores referem-se ao 'dia do seu aniversário' e ao 'pensar em sua mãe', que estão relacionadas com um momento onde, normalmente, a atenção da família e dos amigos está voltada totalmente para ela e por ser celebrado com presentes e comemorações gerando grande satisfação.

Em um estudo realizado por Ferreira, H. S. (2005), com 60 crianças de 4 a 6 anos de idade, também foi encontrada uma grande satisfação das crianças para a questão referente ao 'dia do aniversário'. O autor chama a atenção para o fato de no dia do aniversário, principalmente para crianças de 4 a 6 anos, a grande expectativa é a de receberem presentes, sendo os preferidos, brinquedos.

Fialho (2006) ressalta a importância do brinquedo e do brincar no desenvolvimento infantil. Por meio deles, as crianças vão adentrando no mundo dos adultos e construindo sua própria identidade, seja com brinquedos artesanais ou industrializados.

Torna-se nítido que o domínio família gera grande satisfação. Isso pode estar relacionado ao fato de passar boa parte do seu dia longe de sua família. Por esse motivo tendem a supervalorizar a figura da mãe, resultado semelhante foi obtido na questão 10 que se refere ao momento em que a criança pensa em seu pai.

Outro fator preponderante foi relacionado ao período das férias. Segundo Lima (2008), essa questão está ligada a momentos de lazer, onde as crianças normalmente não possuem uma rotina diária de horários escolares, permitindo que se dediquem exclusivamente às atividades que geram grande satisfação, como é o caso de brincar com os amigos, viajar com os pais ou, até mesmo, assistir televisão.

Outra questão relacionada ao lazer que obteve grande satisfação entre as crianças diz respeito aos momentos de brincadeiras no recreio escolar. Este espaço de tempo, segundo Lopes et al. (2012) é importante no âmbito da promoção de atividade física em crianças por apresentar 
possibilidade de acúmulos ao longo do dia, além de valorizar aspectos sociais; emocionais e cognitivos. É neste momento que a criança entra em um mundo de fantasia, podendo satisfazer seus desejos, sejam de ordem afetiva ou relacionada à estima.

Wajskpo (1995) define o brincar, numa perspectiva sociocultural, como uma maneira que as crianças têm para interpretar e assimilar o mundo, a cultura, os objetos, as relações interpessoais, dentre outras.

Resultados semelhantes foram encontrados no estudo de Verdugo e Sabeh (2002), no qual, ao questionar 199 crianças de 8 anos, de nacionalidade argentina e espanhola, sobre o que faz a criança contente e feliz, $46 \%$ relataram as atividades de ócio e as ações recreativas.

A família novamente torna-se fator determinante para a QV das crianças, pois ao serem questionadas sobre como se sentem ao estar longe de sua família a pontuação foi negativa, o que caracteriza grande insatisfação das crianças ao permanecerem longe dos pais demonstrando fragilidade em relação à autonomia.

Em um estudo realizado com 232 crianças, com idade entre 7 e 12 anos do ensino fundamental da rede pública de ensino, Lima (2008) também caracterizou a questão da ausência familiar como insatisfação por parte da criança. Outros resultados semelhantes foram encontrados no trabalho de Fialho (2006). No estudo foram avaliados um total de 90 crianças, sendo 45 da rede pública e 45 da rede privada, identificando que as crianças da escola pública também apontaram a distância familiar como negativa.

A maior insatisfação demonstrada corresponde ao internamento no hospital. A doença na infância, de um modo geral, é vinculada à imagem de uma experiência negativa (KUCZYNSKI; ASSUMPÇÃO, 1998). Em estudo de Ferreira, D. C. (2005) - com um grupo de pacientes pediátricos infectados pelo Vírus da Imunodeficiência Humana (HIV) - e de Frota et al. (2010) - em crianças com insuficiência renal crônica -, novamente a internação hospitalar foi mais negativa diante do cotidiano das crianças, pois os aspectos que fazem parte do ambiente são insalubres.

As questões relacionadas ao domínio da autonomia apresentaram o menor escore entre as crianças. O resultado pode estar relacionado ao fato das questões referentes a este domínio estarem ligadas a brincar sozinho (questão 15), a dormir fora de casa (questão 17), quando os amigos falam de você (questão 19), estar longe da família (questão 23) e ao receber as notas da escola (questão 24). Tais questões apresentam insatisfação entre as crianças participantes do presente estudo. Resultados semelhantes foram encontrados no estudo de Lima (2008), demonstrando o alto grau de dependência dessa faixa etária aos cuidados dos pais.

Ao analisar o escore geral da percepção de QV, verificou-se os meninos com nível de satisfação maior do que as meninas. Segundo Finco (2004), as diferenças de gênero em crianças demonstram comportamentos, preferências, competências, atributos de personalidade apropriados para o seu sexo, seguindo normas e padrões estabelecidos. De acordo com Vianna e Finco (2009), o papel da cultura nos processos de socialização e de formação de meninas e meninos desde suas primeiras experiências de vida na instituição escolar, podem justificar as diferenças de percepções em relação ao gênero.

Apesar da apresentação de níveis de QV de pré-escolares, este estudo tem limitações metodológicas, sendo desenvolvido com uma quantidade pequena de participantes, de apenas uma região, apresentando, por conseguinte, possibilidades limitadas de generalização dos resultados, além de que, segundo Matza et al. (2004), crianças podem não ser capazes de fornecer informações confiáveis sobre construções relacionadas com a saúde de modo complexo ou abstrato.

\section{Considerações finais}

O estudo procurou compreender de que forma os pré-escolares percebem sua QV e quais os fatores apresentam maior influência. Pode-se perceber que as questões relacionadas aos domínios

R. bras. Qual. Vida, Ponta Grossa, v. 7, n. 2, p. 56-64, abr./jun. 2015. 
de lazer e de família apresentam maior satisfação. No outro extremo, encontram-se as questões relacionadas aos domínios de autonomia e de função apresentando menor nível de satisfação.

Foi identificada a valorização da família, o que indica importância da estrutura familiar na segurança afetiva e social de uma criança em idade pré-escolar. A idade pré-escolar é o momento em que elas saem por um período de casa e precisam aprender a conviver com a figura de professores e de colegas de classe, pessoas até então estranhas no seu cotidiano. Fica, assim, demonstrado o papel fundamental da escola em suprir esses momentos, proporcionando propostas que envolvam o brinquedo e o brincar nas atividades de aprendizagem e, de alguma forma, resgatar a presença dos pais na escola, estimulando a criação de um ambiente educacional saudável contribuindo para uma melhor percepção da QV do pré-escolar.

Vê-se necessário a avaliação da QV infantil, considerando a criança como ator principal, preconizando, assim, sua interpretação sobre o meio, possibilitando diagnósticos de fatores que contribuem para a insatisfação ou a perturbação do processo de desenvolvimento.

\section{Referências}

ASSUMPÇÃO JR., F. B.; KUCZYNSKI, E.; SPROVIERI, M. H; ARANHA E. M. G. Escala de avaliação da qualidade de vida (Autoquestionnaire qualité de vie enfant imagé - AUQEI): validade e confiabilidade de uma escala de vida em crianças de 4 a 12 anos. Arquivos de Neuro-Psiquiatria [online], v. 58, n. 1, p. 119127, 2000. crossef

BARREIRE, S. G.; OLIVEIRA, O. A.; KAZAMA, W.; KIMURA, M.; SANTOS, V. L. C. G. Qualidade de vida de crianças ostomizadas na ótica das crianças e das mães. Jornal de Pediatria, v. 79, n. 1, 2003. crossef

CICONELLI, R. M.; FERRAZ, M. B.; SANTOS, W.; MEINÃO, I.; QUARESMA, M. R. Tradução para a língua portuguesa e validação do questionário genérico de avaliação de qualidade de vida SF-36 (Brasil SF36). Revista Brasileira de Reumatologia, v. 39, n. 3, p. 143-150, 1999.

CONNOLLY, M. A.; JOHNSON, J. A. Measuring quality of life in peadiatric patientens. Pharmacoeconomics, v. 16, p. 605-625, 1999. Crossef

ELIAS, A. V. Autismo e qualidade de vida. 2005. 146f. Dissertação (Mestrado em Ciências Médicas) Programa de Pós-Graduação em Ciências Médicas, Faculdade de Ciências Médicas da Universidade Estadual de Campinas.

FERREIRA, D. C. Estudo de validação de uma escala de qualidade de vida em um grupo de pacientes pediátricos infectados pelo HIV. 2005. 47f. Dissertação (Mestrado em Saúde da Criança e do Adolescente) - Programa de Pós-Graduação em Saúde da Criança e do Adolescente, Universidade Federal Fluminense, Niterói.

FERREIRA, H. S. Percepção sobre qualidade de vida entre crianças de 4 a 6 anos: Educação Física em saúde na escola. 2005. 146f. Dissertação (Mestrado em Educação em Saúde) - Programa de Pós-Graduação em Educação em Saúde, Universidade de Fortaleza.

FIALHO, L. M. F. Qualidade de vida na infância: visão de alunos da rede pública e privada de ensino. 2006. 104f. Dissertação (Mestrado em Educação em Saúde) - Programa de Pós-Graduação em Educação em Saúde, Universidade de Fortaleza.

FINCO, D. Faca sem ponta, galinha sem pé, homem com homem, mulher com mulher: relações de gênero nas brincadeiras de meninos e meninas na pré-escola. 2004. 184f. Dissertação (Mestrado em Educação) - Programa de Pós-Graduação em Educação, Universidade Estadual de Campinas, 2004.

R. bras. Qual. Vida, Ponta Grossa, v. 7, n. 2, p. 56-64, abr./jun. 2015. 
FROTA, M. A.; MACHADO, J. C.; MARTINS, M. C.; VASCONCELOS, V. M.; LANDIN, F. L. P.; Qualidade de vida da criança com insuficiência renal crônica. Escola Anna Nery Revista de Enfermagem, Fortaleza, v. 14, n. 3, p. 527-533, 2010. Crossef

GASPAR, T.; MATOS, M. G.; RIBEIRO, J. L. P.; LEAL, I. Qualidade de vida e bem-estar em crianças e adolescentes. Revista Brasileira de Terapias Cognitivas, v. 2, n. 2, p. 47-60. 2006.

GORDIA, A. P.; SILVA, R. C. R.; QUADROS, T. M. B.; CAMPOS, W. Variáveis comportamentais e sociodemográficas estão associadas ao domínio psicológico da qualidade de vida de adolescentes. Revista Paulista de Pediatria, v. 28, n. 1, p. 29-35, 2010. rossef

KUCZYNSKI, E.; ASSUMPÇÃO JR., F.B. Transtornos psiquiátricos em crianças e adolescentes com câncer. Sinopse de Pediatria, v. 3, p. 56-64, 1998.

LAURENTI, R. A mensuração da qualidade de vida. Revista da Associação Médica Brasileira, v. 49, n. 4, p. 361-362, 2003. rossef

LIMA, M. R. C. Educação para qualidade de vida: a escola e a família como aliados na luta da conscientização. Polêmica Revista Eletrônica, Rio de Janeiro, v. 7, p. 98-104, 2008.

LOPES, L.; SANTOS, R.; LOPES, V.; PEREIRA, B. A importância do recreio escolar na atividade física das crianças. In: CONDESSA, I.; PEREIRA, B.; CARVALHO, G. (Coord.). Atividade Física, Saúde e Lazer. Educar e Formar. Braga: Centro de Investigação em Estudos da Criança, Instituto de Educação, Universidade do Minho, 2012.

MANIFICAT, S.; DAZORD, A. Évaluation de la qualité de vie de l'enfant: validation d'un questionnaire, premiers résultats. Neuropsychiatrie de l'Enfance et de l'Adolescence, v. 45, p. 106-114, 1997.

MATZA, L. S.; SWENSEN, A. R.; FLOOD, E. M.; SECNIK, K.; LEIDY, N.K. Assessment of HealthRelated quality of life in children: a review of conceptual, methodological, and regulatory issues. Value in Health, v. 7, n. 1, p. 79-92, 2004. Crossef

SOARES, A. H.; MARTINS, A. J.; LOPES, M. C.; BRITTO, J. A.; OLIVEIRA, C. Q.; MOREIRA, M. C. Quality of life children and adolescents: a bibliographical review. Ciência \& Saúde Coletiva, v. 16, p. 31973206, 2011. crossef

SOUZA, J. G. S.; PAMPONET, M. A.; SOUZA, T. C. S.; PEREIRA, A. R.; SOUZA, A. G. S.; MARTINS, A. M. E. B. Instrumentos utilizados na avaliação da qualidade de vida de crianças brasileiras. Revista Paulista de Pediatria, v. 32, n. 2, p. 272-278, 2014. Crossef

VERDUGO, M. A.; SABEH, E. N. Evaluación de la percepción de calidad de vida en la infancia. Psicothema, Salamanca, v. 14, n. 1, p. 86-91, jun. 2002.

VIANNA, C.; FINCO, D. Meninos e meninas na educação infantil: uma questão de gênero e poder. Cadernos Pagu, v. 33, p. 265-283, 2009. rossef

WAJSKOP, G. O brincar na educação infantil. Cadernos de Pesquisa, São Paulo, n. 92, p. 63-68, fev. 1995. 
Recebido em: 29 maio 2015. Aprovado em: 28 jun. 2015.

R. bras. Qual. Vida, Ponta Grossa, v. 7, n. 2, p. 56-64, abr./jun. 2015. 